\title{
Clients, usagers et suspects : les mots de la protection sociale en Grande-Bretagne et en France
}

The Vocabulary of Welfare in Great Britain and France

Jean-Paul Révauger

\section{(2) OpenEdition}

12 Journals

Édition électronique

URL : http://journals.openedition.org/rfcb/6142

ISSN : 2429-4373

Éditeur

CRECIB - Centre de recherche et d'études en civilisation britannique

Édition imprimée

Date de publication : 1 juin 2010

ISSN : 0248-9015

\section{Référence électronique}

Jean-Paul Révauger, «Clients, usagers et suspects : les mots de la protection sociale en GrandeBretagne et en France ", Revue Française de Civilisation Britannique [En ligne], XV-4 | 2010, mis en ligne le 01 juin 2010, consulté le 07 janvier 2021. URL : http://journals.openedition.org/rfcb/6142

Ce document a été généré automatiquement le 7 janvier 2021.

\section{(i) $\odot$

Revue française de civilisation britannique est mis à disposition selon les termes de la licence Creative Commons Attribution - Pas d'Utilisation Commerciale - Pas de Modification 4.0 International. 


\title{
Clients, usagers et suspects : les mots de la protection sociale en Grande-Bretagne et en France
}

\author{
The Vocabulary of Welfare in Great Britain and France
}

Jean-Paul Révauger

1 Les discours tenus sur la protection sociale émanent de trois types de sources. Les premières sont institutionnelles, et proviennent soit des organismes créés pour gérer le système, soit des responsables politiques amenés à concevoir, diriger et faire évoluer les politiques publiques en la matière. Les secondes émanent des "experts", commentateurs universitaires ou chercheurs, qu'ils soient employés par un organisme de recherche de type public ou par une ONG. Les auteurs, dans cette catégorie, sont en fait très souvent les mêmes personnes, en particulier en Grande-Bretagne, où la frontière entre la Social Policy Association et le milieu associatif, comme le Child Poverty Action Group, est très poreuse. Le troisième type de sources est constitué par la production des journalistes plus ou moins spécialisés, des écrivains et essayistes révulsés par la pauvreté ou l'oppression, ou même des artistes inspirés par le spectacle de la guenille. Les gestionnaires, les experts et les écrivains s'influencent mutuellement depuis toujours dans le domaine social. L'efficacité des articles de Mayhew ${ }^{1}$, des romans de Dickens ${ }^{2}$ ou du reportage d'Orwell ${ }^{3}$ et de la série télévisée de la BBC Breadline Britain ${ }^{4}$ n'est plus à démontrer. Certains experts accèdent au statut de conseillers du prince, et certains ministres sont issus du milieu associatif5. Néanmoins, devant l'étendue du sujet, cet article se concentrera plus particulièrement sur la première catégorie, les discours institutionnels et politiques. Le parti pris comparatif a pour objectif de faire ressortir les singularités de chaque démarche nationale, tout en prenant la mesure des convergences et, éventuellement, de l'européanisation du débat.

Une démarche axée sur les «mots » ou la stratégie de communication de tel ou tel groupe ou, terme encore plus sujet à caution, la «représentation» de tel ou tel phénomène doit être extrêmement prudente. La parole est par définition sélective, elle est mise en forme, parfois à l'issue d'un processus délibéré, complexe et réfléchi. La 
communication s'est totalement professionnalisée, en particulier en Grande-Bretagne, et plus encore dans les milieux du New Labour que par le passé. Le spin n'est pas une invention de Tony Blair, mais le souci de présenter les politiques publiques sous un jour susceptible d'avoir un impact électoral positif est devenu rapidement, dans les années précédant 1997, un élément essentiel du dispositif. Cette obsession pour la communication est largement due à la conviction qu'avaient les travaillistes d'avoir amplement mérité de remplacer les conservateurs à Downing Street après 11 ans de thatchérisme, et de ne devoir leurs défaites qu'à une piètre maîtrise de leur expression publique, face à des médias hostiles. Cette analyse avait des fondements réels, mais a un peu conduit le New Labour à donner la priorité à la communication sur la substance, suscitant parfois dans le public une réaction de cynisme, pas toujours justifiée. La politique sociale n'a pas échappé à ce phénomène.

Le fait que la communication et la « représentation » soient sélectives doit nous inciter à ne pas confondre réalité d'un modèle social et représentation des questions sociales. En France, la question de la protection sociale ne se résume pas aux campeurs du canal St Martin, aux émeutes de 2005 et à la grippe A, soit aux questions de l'exclusion sociale, de la déréliction du lien social chez les jeunes Français issus de immigration, ou de la gestion des risques sanitaires. L'analyse de la réalité d'un modèle social doit prendre en compte les grandes masses, les évolutions lentes intéressant l'ensemble des citoyens, autant que les crises. Or, la communication publique des politiques entretient avec les médias un lien beaucoup plus subtil que par le passé. L'occupation absolument quotidienne des médias grâce à une succession de « coups » est un élément permettant de dominer le champ politique. La gestion de chaque crise, même si elle est médiocre, permet de renvoyer à l'opinion l'image de gestionnaires compétents et mobilisés, voire pleins d'humanité et compatissants. L'envol des dépassements d'honoraires chez les médecins français n'est pas une nouvelle, mais une intervention présidentielle courroucée pour accélérer le rythme des vaccinations en est une. Les maitres mots sont « crise » et « émotion ».

4 Inversement, la logique des « coups médiatiques » est consubstantielle aux médias, et ne résulte pas seulement de leur manipulation par les professionnels de la «com ». Les médias qui tentent d'échapper à la logique de la mise en scène des crises ne connaissent aucun succès, et ne survivent que s'ils sont financés institutionnellement. Il n'est pas de lecture plus ennuyeuse que celle des bulletins des mutuelles de la santé, quelle que soit, au fond, l'importance de la lutte contre l'ostéoporose ou l'herpès génital, sujets de prédilection de ces organes édifiants. Ce travail est fondé sur la communication institutionnelle et les débats politiques, mais tente d'éviter une simple analyse du social à travers le prisme médiatique. Ces prolégomènes sont donc des mises en garde. L'image que donnent, ou que souhaitent donner, politiques et institutions a un rapport très indirect avec la réalité.

\section{Méthodologie}

Une comparaison entre le Royaume-Uni et la France dans le domaine des «mots de la protection sociale » fait apparaître des différences absolument caricaturales entre les deux pays. La caricature doit être entendue ici non pas comme une image fausse, mais comme l'exagération de tendances bel et bien présentes, voire problématiques. Il faut être donc prêt à intégrer la distorsion entre la réalité et la "représentation». 
Globalement, les termes employés au Royaume-Uni sont liés à des politiques publiques, ceux qui sont utilisés en France à des institutions. Si on voulait forcer le trait, les termes les plus courants résumant les deux approches nationales serait «choice » en anglais et «assujetti » en français. Ceci est d'autant plus intéressant que, en matière de santé, les Britanniques ont un éventail assez limité en termes de choix, alors que l'inverse est vrai de la France, et le degré de contrôle ou l'autorité exercée par la Sécurité sociale française reste tout à fait théorique. Le terme « assujetti » est utilisé ici pour sa valeur symbolique, car, en réalité, il a été très fréquent dans le passé en France, mais ne l'est plus. Il est en revanche toujours d'actualité dans le système belge ${ }^{6}$. Il n'en reste pas moins que le système britannique donne une image dynamique et le système français une image institutionnelle. Les corpus utilisés ici comprennent à la fois les sites web parlementaires et les sites institutionnels de la sécurité sociale et des ministères concernés. Le traitement quantitatif des corpus permet de tester des hypothèses, et non de les faire surgir. L'utilisation de sites comme ceux des assemblées parlementaires ou des grandes institutions, a un double avantage. D'une part, elle permet des comparaisons transnationales entre corpus à peu près comparables. D'autre part, les moteurs de recherche, relativement évolués, permettent un repérage, qui, sans être très satisfaisant, constitue un premier tri. Cependant, il s'agit là d'une utilisation détournée des moteurs de recherche proposés, qui ont pour fonction de retrouver un discours particulier, ou les positions de tel ou tel parlementaire, et non de constituer des séries. Certains termes, très spécialisés et transparents en politique sociale, comme "flexibility» sont repérés par le logiciel en fonction de leur acception courante, et renvoient par exemple à la souplesse prêtée à la politique étrangère britannique plutôt qu'aux caractéristiques du marché du travail. "Occupier» ne renvoie pas nécessairement à "owner occupier ", mais à la Cisjordanie. En outre, la présence d'une thématique, ou d'un concept, ne nous renseigne pas sur la façon dont elle est présentée, qui peut être positive aussi bien que négative. Ce sont les courants eurosceptiques qui parlent le plus de l'Europe, contribuant par là à européaniser le débat politique. Ce ne sont pas les promoteurs des privatisations qui en parlent le plus.

6 La problématique de départ, dans cette recherche, est de trouver la façon dont les usagers des services publics dans le domaine social sont présentés dans le discours officiel, politique ou institutionnel, dans les deux pays, et de faire ressortir des différences a priori considérables. Au delà de l'intérêt anecdotique d'une étude de la « représentation », la façon dont les « usagers » ou les « clients » sont présentés nous donne une indication sur la façon dont ils sont perçus par les concepteurs des politiques sociales. Cependant, il peut y avoir une énorme distance entre l'analyse faite en interne par des décideurs politiques et l'image des «usagers » qu'ils tentent de projeter et d'accréditer. Peut-être, en réalité, les ministres de la Santé français et leurs conseillers ne perçoivent-ils pas les usagers comme des adolescents dépensiers et irresponsables, même s'ils pensent qu'il convient de les morigéner. Il y a donc un rapport fort mais indirect entre l'image des usagers telle qu'elle est projetée et les politiques publiques. Julian Le Grand, économiste de renom jouissant d'une grande influence depuis les années 1990 dans la définition des politiques publiques écrivait en 1997 :

Sometimes, the assumptions concerning motivation and behaviour are explicit; more often they are implicit, reflecting the unconscious values or beliefs of the policy-makers concerned. Conscious or not, the assumptions will determine the way that welfare institutions are constructed. ${ }^{7}$ 


\section{Knights, pawns and knaves}

7 La vision de Julian Le Grand a beaucoup influencé les décideurs. Selon lui, les bénéficiaires et les acteurs de la protection sociale étaient jusqu'aux années Thatcher classés en deux catégories: "knights» et "pawns». Cette vision, qui émanait des penseurs dominant le champ, comme Richard Titmuss' ${ }^{8}$, Anthony Crosland ${ }^{9}$ ou T.H. Marshall ${ }^{10}$, qui appartenaient à la tradition social-démocrate, était de facto partagée par les administrateurs du système, et, jusqu'à l'arrivée de Keith Joseph ${ }^{11}$, par les Conservateurs paternalistes de la tradition "One Nation». Le Grand insiste sur la vision éthique d'un Titmuss, et à juste titre, sur l'ouvrage qui est le plus emblématique de la période, The Gift Relationship, dans lequel Titmuss, notre expert en techniques prudentielles devenu moraliste, explique qu'il est essentiel d'offrir à l'homme des occasions de se comporter de façon conforme à l'éthique, et de cultiver cette vertu, par exemple en étant donneur de sang. Le système repose donc sur une vision positive, optimiste, de l'humanité. En pratique, cependant, les nobles chevaliers qui administrent la protection sociale avec conscience et compétence traitent le public comme des pions.

Patients were expected to live up to their appellation and be patient. They were to wait patiently in queues at GP's surgeries [...] If they needed further treatment, they were to wait for their turn on hospital waiting lists. When the time arrived for them, they were supposed cheerfully to accept being on a public ward, being served horrible food and, most significantly, being treated by doctors too busy, or too elevated, to have time to explain what was happening to them. ${ }^{12}$

8 Cette description qui correspond à peu près au sort réservé aujourd'hui à la grande majorité des êtres humains dans les hôpitaux publics de par le monde, quand ils ont la chance de parvenir à un hôpital, aurait pu être écrite par un conservateur britannique ou par un médecin libéral français. Il n'en n'est rien, Le Grand était le promoteur de la notion de "quasi marché ». Selon lui, la Grande-Bretagne avait basculé au cours des années 1980 et 1990 dans une autre problématique, dans laquelle patients et acteurs sont perçus comme des "knaves ", c'est-à-dire des agents économiques rationnels et conscients, utilisant délibérément toutes les possibilités offertes par le système pour maximiser leurs gains et avancer leur intérêts. En fait, derrière la figure du " knave » se trouve l'individu, agent indépendant, tel qu'il est présenté par les libéraux, au sens premier du terme, à la fois économique et philosophique. Il n'est pas mû par des considérations éthiques ayant un lien avec une autorité transcendantale aussi impérieuse que mystérieuse. Il n'est pas spontanément obéissant et soumis à l'autorité. Les conservateurs lui prêtent une rationalité qui lui fait beaucoup d'honneur, à partir des années 1970, et fait la fortune de la "rational choice theory $\|^{13}$. Ce n'est donc ni un "knight» ni un "pawn». Dans cette vision des choses, on passe donc directement du statut de sujet de Sa Majesté ou de moine vertueux à celui d'agent économique, producteur ou consommateur, sans que la dimension politique soit présente. Le citoyen et le travailleur organisé dans une logique mutualiste, concrétisée par l'assurance sociale dans les systèmes d'origine corporatiste, sont un peu les chaînons manquant dans cette vision très britannique et très économique de la société. En revanche, ceci permet de comprendre comment la vision thatchérienne du monde a pu non seulement dominer provisoirement le champ, mais imposer sa marque durablement, y compris sur le travaillisme. En effet, même si la dimension éthique est importante en Grande- 
Bretagne, elle ne saurait concurrencer la logique économique. Dans la meilleure des configurations, elle peut être en communion avec elle.

\section{To Market ! To Market !}

9 Le New Labour met donc en avant l'individu, dont les désirs doivent être des ordres, comme ceux du client roi. Aussi, le terme de «choice» devient-il le «buzz word» central, autour duquel s'organise toute la rhétorique. Ceci apparaît clairement sur le site parlementaire. Au terme "NHS», le mot "market» est associé 17441 fois, le terme "choice» 9747 fois, contre 14376 pour « equality ». Les usagers du National Health Service (NHS) sont des «users» dans 6442 fichiers, mais des «customers» 10948 fois, contre 23673 pour «patients». Ceci est d'autant plus significatif que le NHS est en situation de quasi monopole, pour ce qui est des utilisateurs, même si l'offre de soins inclut maintenant des prestataires privés, par le biais des PPP ${ }^{14}$. Les termes de «market » et de "customers" sont donc des exemples parfaits de "fausse conscience», ou du moins révèlent un parti pris idéologique intéressant. Le site officiel du NHS est d'ailleurs intitulé «NHS Choices » ${ }^{15}$, ce qui accorde à ce terme une place véritablement centrale ${ }^{16}$.

A priori, le contraste avec la France est saisissant. Le terme "choix ", sur le site de l'Assemblée nationale, consulté en janvier 2010 pour une période couvrant la dernière législature, se retrouve associé à la santé 2110 fois, mais dans l'acception «santé publique ", ce qui dénote une option en fait totalement contraire à celle qui est impliquée par la logique du « choix » individuel. Il est associé à « éducation » 1215 fois, ce qui est peu, à «traitement médical » 413 fois, ce qui est minime. La plus grande occurrence du terme est lorsqu'il est associé à "établissement ", mais, dans ce cas, il renvoie en pratique à des situations extrêmement variées, dans le domaine médical comme éducatif, mais aussi pénitentiaire, ce qui ne manque pas de sel, l'incarcération étant par définition la négation du choix. Le terme beaucoup plus marqué idéologiquement car clairement militant de «libre choix » est employé 891 fois, ce qui est significatif pour une expression aussi chargée. En réalité , un sondage parmi les sites repérés par le moteur de recherche permet de constater que le terme est employé dans des domaines extrêmement variés, et fait référence au vote des députés, au pouvoir des consommateurs, et à la protection sociale, mais essentiellement en matière de solution de garde pour les enfants en âge pré-scolaire ${ }^{17}$.

11 Loin d'enfoncer des portes ouvertes et de confirmer des choses que l'on sait déjà, le sondage quantitatif, même aussi primitif que celui qui peut être fait en utilisant des outils conçus pour un autre usage, fait apparaître un décalage important entre la réalité et la «représentation». Le système de santé britannique est parmi les plus démarchandisés du monde, pour utiliser la terminologie d'Esping Andersen ${ }^{18}$. L'usager ne se voit pas offrir la possibilité d'acheter un service de qualité différente en fonction de son budget, contrairement à ce qui est le cas dans les systèmes duals de type étatsunien. Certes, au niveau de l'offre de soins, les pouvoirs publics britanniques ont pris une option qui peut à terme rapprocher le système britannique du système français. En invitant le secteur privé à investir dans l'offre de soins, moyennant la garantie d'une rémunération sans risque, car offerte par l'État, les promoteurs des PPP tournent le dos à la tradition britannique d'un monopole public de l'offre de soin. Ceci présente l'avantage d'améliorer rapidement l'offre de soins sans grever le budget de l'année, mais au prix d'un endettement qui pèsera pendant des décennies sur le budget de la 
santé, et sur le contribuable. La Grande-Bretagne s'apprête donc à importer le déficit de la sécurité sociale, spécialité française dont on ignorait les attraits. Cependant, pour l'usager britannique, l'opération est, sur le court terme, non seulement indolore, mais positive. L'accès aux soins reste gratuit pour l'individu, et l'éventail des possibles, pour utiliser la rhétorique des partisans de la liberté de choix, s'ouvre largement. Les waiting lists, qui, effectivement, étaient un vrai problème, ont donc été réduites - grâce au recrutement de personnels de santé par le NHS en premier lieu, mais aussi, parfois, grâce à l'achat par le NHS de services médicaux privés, y compris en faisant franchir la Manche à des malades britanniques soignés dans des cliniques privées françaises aux frais du NHS.

L'ambiguïté fondamentale du terme de "choice» apparaît donc clairement ici. Les Britanniques emploient délibérément un terme associé au marché, à la fois pour faire pénétrer peu à peu la logique de marché dans les mentalités et dans le secteur public, en bonne logique thatchérienne et post-thatchérienne, et pour "jouer à la marchande » dans un système de santé public et "free at the point of delivery ». Le " quasi marché » cher à Julian Le Grand est donc ... un marché de dupes. Chacun a en mémoire, dans les années 1990, le jeu de Monopoly de l'Internal Market, voyant les General Practitioners dotés de pouvoirs budgétaires, acheter avec de l'argent public des services à des organismes publics, destinés à soigner gratuitement des patients, aux frais de la nation. Il est évident que les patients britanniques de 2010 se voient offrir davantage de " choix " par exemple en matière de lieu d'hospitalisation ou de " practice», mais que le NHS reste un service public de santé universaliste et très peu inégalitaire. Moins on a le choix, plus on en parle.

Inversement, la France a vu évoluer son propre système dans un sens opposé. Le déremboursement de certains médicaments, l'existence d'un système de mutuelles ou d'assurances privées complémentaires, présentant au public une offre de plus en plus diversifiée, et creusant le fossé entre assurés sociaux, la co-existence d'établissements privés et publics offrant souvent des prestations de qualité très différente, les dépassements d'honoraires systématiques dans certaines villes, le refus de beaucoup de médecins d'accepter de soigner les bénéficiaires de la couverture maladie universelle (CMU), ont très sérieusement entamé le caractère universel et égalitaire de la Sécurité sociale. Le système français est donc beaucoup plus "marchandisé", soumis à la capacité des individus de solvabiliser leur demande, que le système britannique, même si la France et la Grande- Bretagne font toujours partie du modèle européen, largement démarchandisé. Le " choix » y est donc maximal, pour peu que les individus soient en mesure de l'assumer financièrement. L'état de délabrement de l'une des principales conquêtes de la Libération n'est pas un mince paradoxe, d'autant que le législateur avait pris l'option de confier la gestion, pour une large part, aux partenaires sociaux. Les modifications introduites au début de la Cinquième République, à la faveur des ordonnances décrites par les syndicats de l'époque comme "scélérates", expliquent sans doute la paralysie du mouvement social français devant cette évolution ${ }^{19}$. Le rôle des mutuelles a également connu des changements importants. Issues des rangs du salariat, elles se sont transformées peu à peu en sociétés d'assurance dépolitisées, voire en auxiliaires de l'État.

Si un « quasi marché » existe et progresse quelque part en Europe, c'est bien en France, et non chez MM. Blair et Brown. Néanmoins, la rhétorique du choix y est marginale. Le vocabulaire dominant, dans la communication publique de la Sécurité sociale, est celui 
de l'autorité et du paternalisme. L'assuré social, qui est censé être un citoyen, un mutualiste, et un travailleur cogérant les caisses, est avant tout un sujet, passif, soupçonné d'irresponsabilité financière, à qui il convient de mettre un peu de plomb dans la cervelle. La célèbre campagne de communication sur les antibiotiques, lors de laquelle un jeune enfant répétait avec une voie de fausset lancinante que "les antibiotiques, c'est pas automatique ", était assez emblématique. L'assuré social est un mineur, sympathique et qui retient vite sa leçon, pour peu qu'on la lui répète assez souvent. Une visite sur le site de la Sécurité sociale et dans les utiles glossaires qu'il contient est révélatrice ${ }^{20}$. Outre une élucidation des centaines de sigles mystérieux qui font le charme de notre système, on y trouve une délicieuse succession de termes comme «action civile, affiliation, avenant, avis d'imposition, ayant droit, code pénal, confiscation, conformité $»^{21}$. Il faut avant tout faire en sorte que les cotisations soient réglées, souci bien compréhensible dans la logique institutionnelle française. En termes de communication externe et de "représentation", la "Sécu» a donc plusieurs décennies de retard sur la Grande-Bretagne, qui parvient à persuader ses patients que ce sont des clients, et que le menu unique est une carte. Certes, les « assujettis » ont discrètement disparu du vocabulaire français, victimes de la vigilance de quelque membre de l'Inspection Générale des Affaires Sociales, ou de quelque autre grand commis de l'État. Néanmoins, le vocabulaire institutionnel, juridique, administratif et les paternelles exhortations à la modération et à la sagesse dominent. Cela ne signifie bien sûr nullement que la protection sociale de notre pays soit régie par le principe d'autorité. Il n'en est rien, évidemment. La question de la légitimité et de l'étendue du pouvoir médical est distincte de celle de l'autorité, qui, ici, s'entend du point de vue du rapport de l'individu à l'État.

\section{Choix et stratégie de responsabilisation}

15 La question du choix est intimement liée à celle de la responsabilité individuelle. Elle a, en Grande-Bretagne, une dimension éthique importante. La question de savoir si l'individu est un " pawn», un "knight » ou un " knave » nous amène directement à celle de la fraude, une obsession britannique traditionnelle. Il est rapidement entendu que tous les individus ne sont pas des «knights » vertueux. Or, la question de la fraude est liée à la théorie du choix rationnel. L'individu, en toutes circonstances, va agir en fonction de son intérêt et ne sera pas nécessairement guidé par l'éthique. L'existence de la fraude démontre donc, aux yeux des courants conservateurs qui la mettent au premier plan à partir des années 1970, que le présupposé de Titmuss est faux. Les individus agissent de façon autonome, ils ne se satisfont pas de ce que l'État leur offre, et, s'ils ne sont pas canalisés, ils ont tendance à mettre leur énergie au service d'une mauvaise cause et à voler la collectivité. Les allocations sont des effets d'aubaine, et d'aucuns se complaisent dans le poverty trap, dont la douceur n'est pourtant pas proverbiale. En revanche, en bonne logique smithienne, si l'égoïsme individuel se déploie dans le cadre d'une concurrence pure et parfaite, et encadrée par la loi, alors, il débouchera sur l'excellence. Le fraudeur est donc un agent économique à qui on n'a pas donné de repères. C'est la nécessité d'encadrer les individus et de réduire leur propension à nuire, en permettant à leur ambition de s'exprimer, qui fournit au conservatisme britannique un de ses principes de base. Libéralisme et autorité vont donc de pair. Le New Labour a repris à son compte le discours sécuritaire et méfiant des conservateurs, en s'inspirant, paradoxalement, des écrits du courant communautarien 
des années 1980 sur les droits et les responsabilités ${ }^{22}$. Il ne s'agit donc pas simplement d'une conversion au thatchérisme, à but étroitement électoraliste, mais d'une vision éthique visant à établir la cohésion sociale par le biais d'une culture partagée - de gré ou de force. Il y a de la ferveur évangélique dans certains sermons de Frank Field ${ }^{23}$, et on est plus proche du modèle scandinave, partageant des valeurs fortes et faisant de la surveillance réciproque une vertu, que d'une dictature méditerranéenne.

Or, le « choix » donné aux individus, dans un cadre fixé par l'État, a pour but non pas de les laisser donner libre cours à leur imagination débridée, mais de leur permettre de vivre joyeusement, et de façon ordonnée, leur identité de sujets économiques. Le choix donné aux locataires du secteur public de devenir propriétaires, ou de réguler le comportement de leurs voisins, a un objectif. Il s'agit de rendre les Britanniques « house proud» - terme intraduisible. Les autorités partent du principe que seuls des locataires impliqués dans la gestion de leur quartier, seuls des petits propriétaires pourront réduire les incivilités, lutter contre les papiers gras, les graffitis, le tapage nocturne. Dans un article d'une grande finesse, Greener et Powell font justement remarquer que le terme de choix, promu au rang de slogan, correspond en fait à des réalités très différentes selon les secteurs ${ }^{24}$. En matière de logement, donner le choix, c'est avant tout responsabiliser, faire de l'individu un "stakeholder", autre mot fétiche intraduisible. En revanche, en matière de santé ou d'éducation le terme a une autre fonction. Il s'agit bien ici de social engineering, de modifier le comportement des individus afin de le rendre conforme à la fois à l'éthique et à l'intérêt bien compris du pays. La sinistre figure du (jeune) délinquant est visible en contrepoint derrière celle du bon citoyen, faisant les bons choix en toute lucidité, et appelant le 999 sans arrièrepensée quand besoin est. Les quartiers, appelés sous le New Labour " community ", voire, sans ambiguïté superflue, "parish», sont dotés de pouvoirs nouveaux. Donner les choix au citoyen, c'est se livrer à une opération de "empowerment ", selon la terminologie d'origine américaine. Or, en matière de logement et de gestion des questions sociales dans un quartier, le "empowerment» est une forme de régulation, dans lequel "les technologies de l'autogestion sont utilisées pour réguler les comportements sociaux $»^{25}$. Les Parish Councils ont été réactivés, et des associations de propriétaires, les Common Interest Developments se voient accorder un rôle nouveau - un pouvoir, une possibilité de faire des choix - en matière d'ordre public, de gestion des loisirs, d'aménagement voire de gardiennage. Sans aller jusqu'aux très nombreuses gated communities américaines et aux ghettos dorés d'Afrique du Sud, loin des villes noires ${ }^{26}$, ces enclaves sont de plus en plus coupées du reste de la nation. Il n'est pas évident que « laisser le choix » ou « donner le pouvoir" aux habitants de tel ou tel quartier soit en fin de compte créateur de cohésion au plan national. La signature de « contrats de bon voisinage » et la possibilité pour des résidents de demander au juge l'édiction d'un ASBO (Anti Social Behaviour Order) contre un voisin ou une bande de jeunes ${ }^{27}$ sont bien dans l'ancienne logique de la neighbourhood watch, pratique qui consistait à inciter les résidents d'un quartier à signaler les suspects, et à espionner leur prochain avec l'approbation de la police.

La promotion du « choix » est donc parfois plus une affaire de contrôle social par « la grande famille du maintien de l'ordre ${ }^{28}$ que d'extension des libertés, dans un pays qui est parmi les plus surveillés du monde. 


\section{Conclusion} cours des années New Labour. La figure de la victime ou de l'allocataire a cédé la place à celle de l'individu incité à faire des choix et à assumer les devoirs qui accompagnent les droits. On peut noter un décalage avec le discours tenu par l'Union européenne, qui persiste à cibler certaines catégories touchées par les risques sociaux (pauvreté, lutte contre les discriminations, femmes) en même temps qu'elle prône le développement du " capital humain ». En pratique, en Grande-Bretagne ces " choix » positifs offerts aux individus sont de nature très différente selon les secteurs. En matière de santé, l'éventail n'est pas très large, et les prestations restent de même type, même si des options sont effectivement présentées. L'acte médical sera le même, quel que soit l'hôpital choisi.

Cependant, l'insistance mise par les autorités à inciter les individus à "choisir » déforme dans une large mesure la réalité de l'offre en matière de «social ». Dans des secteurs comme la santé et l'éducation primaire et secondaire la Grande-Bretagne garde un système non "marchandisé ", globalement universel et gratuit. Donner des choix dans le cadre d'un service public gratuit, au nom de valeurs appartenant à la sphère du marché, comme les bienfaits de la concurrence ou la souveraineté du « client/usager » est une curieuse synthèse rhétorique entre le « modèle américain » et l'économie sociale de marché. Il est clair que les autorités impriment fortement leur marque idéologique sur les représentations, et que celles-ci sont guidées par les politiques publiques.

En France, la succession des crises médiatisées et des interventions héroïques tentant de les juguler est la marque de fabrique distincte des pouvoirs politiques. Toutefois, la variété des crises et des champs concernés, la succession des petites phrases assassines et les rivalités personnelles ne permettent pas de donner à l'action politique un sens aussi cohérent que dans le cas du New Labour, qui a fait de la discipline en matière de communication un principe avec lequel on ne transige pas. Seule la thématique du maintien de l'ordre public est une constante en France. Les institutions, en revanche, cherchent à échapper à la politique et projettent une image très administrative, dans laquelle compétence et respect du règlement sont les maîtres mots. Les enjeux politiques considérables liés à l'évolution du système dans le sens de la marchandisation et à la bombe à retardement du financement de la Sécurité sociale sont soigneusement évités, même par le secteur mutualiste, ce géant assoupi, dont l'égalité sociale était pourtant la raison d'être.

\section{BIBLIOGRAPHIE}

ANDERSEN, Gosta Esping. The Three Worlds of Welfare Capitalism. Cambridge: Polity Press, 1990.

BECKER, Gary. The Economic Approach to Human Behaviour. Chicago: Chicago University Press, 1978.

Revue Française de Civilisation Britannique, XV-4 | 2010 
CARRÉ, Jacques \& RÉVAUGER, Jean-Paul (dir.). Écrire la pauvreté. Paris : L'Harmattan, 1995. EDWARDS, John \& RÉVAUGER, Jean-Paul ( eds.). Discourse on Inequality in Britain and France. London: Ashgate, 1998.

FIELD, Frank. Reflections on Welfare Reform. London: Social Market Foundation, 1998.

FIELD, Frank \& OWEN, Matthew. Beyond Punishment : Hard Choices on the Road to Full Employability. London: Institute of Community Studies, 1994.

FLINT, John. «Stratégies d'Empowerment' et lutte contre les comportements 'antisociaux' au Royaume-Uni », Géographie, Économie et Société, vol. 8, n¹, Janvier-Mars 2006, pp. 17-36.

GLENNERSTER, Howard \& LE GRAND, Julian. 'The Development of Quasi Markets in Welfare Provision in the UK', International Journal of Health Services, ${ }^{\circ} 25,1995$, pp. 203-218.

GREENER, Ian \& POWELL, Martin. 'The Evolution of Choice Policies in UK Housing, Education and Health Policy', Journal of Social Policy vol. 38, Part 1, 2008, pp. 63-81.

KVIST, Jon \& SAARI, Juho (eds). The Europeanisation of Social Protection. London: Policy Press, 2007.

LE GRAND, Julian. Equity and Choice: An Essay in Economics and Applied Philosophy. London: Harper \& Collins, 1991.

LE GRAND, Julian. 'Knights, Knaves or Pawns? Human Behaviour and Social Policy', Journal of Social Policy, vol. 26 , Part 2, April 1997, pp. 149-169.

MAYHEW, Henry. London Labour and the London Poor. London: The Morning Chronicle, 1849.

MARSHALL, T.H. Social Policy. London: Unwin Hyman, 1965.

ORWELL, George. Down and Out in Paris and London. London: Victor Gollancz, 1933.

PORTONNIER, Jean-Claude. Glossaire bilingue de la protection sociale. Les termes français. Paris : MIRE, IGAS, 1998.

RÉVAUGER, Jean-Paul. 'Translating Social Policy Concepts : Theory and Practice', International Journal of Social Research Methodology, vol. 14, n, October 2001).

RÉVAUGER, Jean-Paul. «Interprétations culturelles de la pauvreté et stratégies politiques ». In RÉVAUGER, Jean-Paul. (dir.), Pauvreté et inégalités en Grande-Bretagne. Paris : Éditions du Temps, 2000.

RÉVAUGER, Jean-Paul. «La pauvreté, entre réalité objective et stratégies politiques ». In Pauvreté et inégalités en Grande Bretagne, Textes rassemblés par A. CAPET, P. ROMANSKI et A. SY-WONYU. Rouen : Publications de l'Université de Rouen, 2001.

RÉVAUGER, Jean-Paul. 'The Welfare State in France', in ASPALTER, Christian (ed.). Welfare Capitalism Around the World. Taiwan: Casa Verde, 2003.

RÉVAUGER, Jean-Paul. 'Social Policy and the Challenge to the Republican Model'. In COLE, Alistair \& RAYMOND, Gino (eds.). Redefining the French Republic. Manchester: Manchester University Press, 2006, pp. 117-134.

SPICKER, Paul \& RÉVAUGER, Jean-Paul (dir.). Glossaire bilingue des termes anglais de la protection sociale. Paris : MIRE, Ministère du Travail, 1998.

TITMUSS, Richard M. The Gift Relationship. London: Allen \& Unwin, 1971.

TITMUSS, Richard M. Social Policy. London: Unwin \& Hyman, 1974. 


\section{Sites Internet}

Site de la BBC : Breadline Britain.

http://news.bbc.co.uk/2/hi/in_depth/business/2005/breadline_britain/default.stm.

Site de l'Assemblée nationale. http://www.assembleenationale.fr/

Site du NHS «Choices ». http://www.nhs.uk/Pages/HomePage.aspx

Site du parlement britannique. http://www.parliament.uk/index.cfm

Portail de la Sécurité sociale : http://www.securite-sociale.fr/index.html

Portail de l'URSSAF, lexique du recouvrement.

http://www.urssaf.fr/general/outils/glossaire/abcd_01.html.

\section{NOTES}

1. London Labour and the London Poor, 1849.

2. Oliver Twist, 1837.

3. Down and Out in Paris and London, 1933.

4. http://news.bbc.co.uk/2/hi/in_depth/business/2005/breadline_britain/default.stm

5. Frank Field, Martin Hirsch, Fadela Hamara...

6. Les amateurs de culture populaire pourront utilement consulter la bande dessinée «Les Assujettis sociaux », de Christian Binet. Série Les Bidochon, Fluide Glacial, 1985.

7. Julian LE GRAND, 'Knights, Knaves or Pawns? Human Behaviour and Social Policy', Journal of social policy, 26 (2) April 1997, p. 154.

8. The Gift Relationship, 1971. Social Policy, 1974.

9. Antony CROSLAND, The Future of Socialism, London, 1956.

10. Social Policy, 1965.

11. Keith Joseph parvint à la direction du Parti conservateur après la chute d'Edward Heath en 1974 et intégra dans le discours conservateur britannique la thématique d'origine états-unienne sur la culture de la pauvreté. Il ouvre la voie au thatchérisme.

12. 'Knights, Knaves or Pawns ? Human Behaviour and Social Policy', op. cit., p. 156.

13. Gary BECKER, The Economic Approach to Human Behaviour, Chicago University Press, 1978. La rational choice theory consiste à appliquer à l'ensemble des activités humaines les logiques de maximisation des gains qui inspirent certains économistes. Elle participe de cette entreprise de domination de la pensée sociale et politique par la logique économique.

14. Public-private partnership / Partenariat public-privé.

15. http://www.nhs.uk/Pages/HomePage.aspx

16. Site de la Chambre des Communes : http://www.parliament.uk/search/search.aspx

17. http://www.assembleenationale.fr/

18. Gosta Esping ANDERSON, The Three Worlds of Welfare Capitalism, Cambridge: Polity Press, 1990.

19. Dans le projet initial, les caisses étaient gérées par les élus des assurés sociaux, et donc par les syndicats. L'intervention du patronat et le «paritarisme» furent imposés par l'ordonnance Jeanneney du 27 août 1967.

20. Portail de la Sécurité sociale : http://www.securite-sociale.fr/index.html

21. Portail de l'URSSAF, lexique du recouvrement (2 janvier 2010): http://www.urssaf.fr/ general/outils/glossaire/abcd_01.html 
22. Amitai ETZIONI, The New Golden Rule : Community and Morality in a Democratic Society, New York: Basic, 1997.

23. Frank FIELD, Beyond Punishment : Hard Choices on the Road to Full Employability, London Institute of Community Studies, 1994. Reflections on Welfare Reform, London Social Market Foundation, 1998. 24. Ian GREENER \& Martin POWELL,'The Evolution of Choice Policies in UK Housing, Education and Health Policy', Journal of Social Policy, 38, 1, pp. 63-81.

25. John FLINT, «Stratégies d'Empowerment' et lutte contre les comportements 'antisociaux' au Royaume-Uni », Géographie, Économie et Société, vol. 8, n 1, janvier-mars 2006, p. 20.

26. Voir la thèse de Guillaume PLOUGOULM, Citoyenneté et espace: développement, urbanisme et culture politique dans la métropole de Durban (1996-2006). Direction: Jean-Claude Redonnet. Université Paris IV, 2008.

27. John FLINT, «Stratégies d'Empowerment' et lutte contre les comportements 'antisociaux' au Royaume-Uni », op. cit., p. 31.

28. Ibid., p.22.

\section{RÉSUMÉS}

Une analyse sémantique quantitative modeste des sites parlementaires et institutionnels consacrés à la protection sociale en Grande-Bretagne et en France permet de faire ressortir des lignes de force. Ces dernières fournissent des indications sur les stratégies de communication des décideurs plus que sur la réalité. En Grande-Bretagne, la représentation du social a changé très significativement au cours des années New Labour. La figure de la victime ou de l'allocataire a cédé la place à celle de l'individu incité à faire des choix, et à assumer les devoirs qui accompagnent les droits. La notion de choix est mise en avant même dans les secteurs où, en réalité, le choix du citoyen usager est très limité, par exemple dans le système de santé qui, en, Grande-Bretagne, reste universel et égalitaire. Dans le secteur du logement, le choix laissé aux citoyens se conformant à la norme est un élément de contrôle social. Le décalage entre réalité et représentation est donc à la fois considérable et significatif en termes idéologiques. En France, c'est plutôt une image institutionnelle et administrative, voire bureaucratique qui est renvoyée, en décalage avec les enjeux des débats relatifs à la protection sociale.

Even a rather primitive quantitative processing of the vocabulary used by welfare institutions and national Parliaments leads to significant results. At the very least, they highlight differences in official communication strategies. In Britain, the presentation of social issues has changed significantly under New Labour. The deserving claimant no longer occupies centre stage. He has been replaced by an autonomous individual, who is encouraged to make choices, and accept the duties that go with them. Choice is promoted even in sectors where it is of low relevance, such as the NHS, which remains universal, egalitarian, and free at the point of delivery. In the field of housing, choice is mostly exerted by stakeholders who conform to agreed rules, and is an element of social control. The gap between reality and 'representation' is therefore extremely wide, which is probably significant in terms of official strategies. In France the welfare system is typically described in institutional, administrative terms, sometimes bordering on the bureaucratic, which hardly relate to the current debates on the future of social protection. 
AUTEUR

JEAN-PAUL RÉVAUGER

UMR Europe, Européanité, Européanisation, Université de Bordeaux 